\title{
Characterization of Polarization Diversity at the Mobile
}

\author{
Tim W. C. Brown, Member, IEEE, Simon R. Saunders, Member, IEEE, S. Stavrou, Member, IEEE, \\ M. Fiacco
}

\begin{abstract}
Two new models to characterize polarization diversity at the mobile are presented and discussed. The first model builds upon existing theoretical models at the base station to demonstrate how those principles apply to the mobile fading environment. Due to the limitations of antennas at the mobile, this extended model, though relevant, is not sufficient to characterize polarization diversity of the antennas so this paper moves on to show how a new approach to polarization diversity at the mobile exists as an inherent feature within an angular diversity system. Results show that, for two polarizations in a fading environment, the best overall output occurs with a $\pm 45^{\circ}$ element configuration although the diversity combiner itself has optimum performance with a $\pm 67.5^{\circ}$ configuration due to lower correlation at the input. The second model is also used to demonstrate how differently polarized dipoles are using polarization diversity inherent within an angular diversity system.
\end{abstract}

Index Terms - Cross-polar ratio, correlation, branch difference, efficiency loss, antenna diversity

\section{INTRODUCTION}

A NTENNA diversity has been moving to polarization diversity in recent times to mitigate multipath fading as opposed to using the conventional spatial diversity. At the base station this reduces the size of the antenna considerably to alleviate its environmental impact [1-4]. At the mobile terminal, polarization diversity also has a role since the antennas are close together and spatial diversity alone is rarely practical. The most notable work done previously on polarization diversity comes from Vaughan [2] who considers the rotation of two perpendicular antennas and also Kozono [3] who looks at the angular spacing of the antennas symmetrically around the vertical axis. Theory shown in both of these publications is invaluable to apply to polarization diversity of the fading environment at the mobile while taking into account factors not present at the base station. The most

Manuscript received May 3, 2004. This work was supported by Nokia Mobile Phones and the UK Engineering and Physical Sciences Research Council (EPSRC)

Tim W. C. Brown is with the Centre for Communication Systems Research, University of Surrey, Guildford, Surrey, GU2 7XH, UK (email: tim@brown76.name)

Simon R. Saunders is a visiting professor to the University of Surrey, UK.

Stavros Stavrou is with the University of Surrey, UK.

Mauro Fiacco was with the University of Surrey, UK and is now with ip.access Ltd, UK. important additional factors in the mobile case are the wider angle of arrival and also the imperfections in the antennas used to measure the polarization diversity. When evaluating polarization diversity of two mobile terminal antennas, it is important to distinguish the difference between diversity of two mobile terminal antenna polarizations and two polarizations in space. For mobile terminal antennas, the polarization diversity is inherent within an angular diversity system and, as such, needs to be evaluated in terms of polar coordinate antenna polarizations, $A_{\theta}$ and $A_{\phi}$ which are not considered by other authors to date.

This paper therefore presents a new theory to calculate the complex and envelope correlation coefficients, $\rho_{12}$ and $\rho_{e}$, the mean branch power ratio, $k$ and the overall signal compared to a single reference branch, $L$. These new models are built upon existing models applied to the base station in section II and followed by a note in section III concerning the measurement of polarizations at the mobile with application to this theoretical model. Section IV moves onto showing how a completely new approach, first described in [16] is necessary to show that polar coordinate polarization diversity is an inherent feature within an angular diversity system.

As for the diversity combining system, a switched diversity combiner is the only one that can, at present, be economically implemented at the mobile terminal with a single receiver. Consequently selection diversity is considered in this publication, which is the ideal case for a switched combiner. Results in section II show that for the fading environment, the optimum performance of a selection combiner is around the $\pm 67.5^{\circ}$ configuration with minimum correlation, $\rho_{12}$, and unity branch power ratio, $k$. However, due to this being offset by the resultant loss, $L$, compared to a single branch, the optimum output or system gain as explained in this paper is greatest at the $\pm 45^{\circ}$ configuration. Section IV compares the two models presented in this paper to show how there is a difference between the polarization diversity of the fading environment and the polarization diversity of the antennas inherent within an angular diversity system. 


\section{THEORETICAL MODEL FOR THE FADING ENVIRONMENT}

Diversity performance of a polarization diversity system for the fading environment depends on the polarization configuration of two polarizations in space. At this point it is important to note that we are not considering the polarizations of the antennas but the polarization of the signals received in the fading environment - i.e. two polarizations in space, just like two points in space for spatial diversity of a fading environment. It is necessary in this situation to combine both Vaughan's [2] and Kozono's [3] theories together to derive a theory to be applied at the mobile terminal, since both rotation and angular spacing are significant in this case while also the three dimensional angle of arrival is of great importance. Four angles need to be defined here, all illustrated in Figure 1, namely:

- $\quad \alpha$ - The angle of rotation of the polarization relative to the vertical field, $E_{x}$, and the horizontal field, $E_{y}$.

- $\quad \beta$ - The azimuth angle relative to the normal to $E_{x}$ and $E_{y}$

- $\quad \gamma$ - The elevation angle relative to the normal to $E_{x}$ and $E_{y}$

- $\Omega$ - The angular spacing between the two polarization branches in the direction of the vertical axis.

Angles $\Omega$ and $\beta$ are considered by Kozono and $\alpha$ is considered by Vaughan; $\gamma$ is considered by neither. Having established the relevant angles, it is possible to derive the voltages induced in the two antennas. In equation (1) the open circuit voltages, $V_{1}$ and $V_{2}$, are represented as a vector, $\mathbf{V}_{E}$, which do not contradict that of Vaughan and Kozono but must be represented this way for the mobile terminal application as there is a wider angular spread and there are many polarizations.

$$
\mathbf{V}_{E}=\left(\begin{array}{l}
V_{1} \\
V_{2}
\end{array}\right)=\left(\begin{array}{cc}
a e & b f \\
c e & d f
\end{array}\right)\left(\begin{array}{l}
E_{x} \\
E_{y}
\end{array}\right)
$$

where:

$$
\begin{aligned}
a=\sin \left(\alpha+\frac{\Omega}{2}\right) & c=\sin \left(\alpha-\frac{\Omega}{2}\right) \\
b=\cos \left(\alpha+\frac{\Omega}{2}\right) & d=\cos \left(\alpha-\frac{\Omega}{2}\right) \\
e=\cos \beta & f=\cos \gamma
\end{aligned}
$$

Both Vaughan and Kozono represent the E-fields as real Gaussian Rayleigh signals, $r_{1}$ and $r_{2}$, with a uniform phase distribution $\phi_{1}$ and $\phi_{2}$ respectively [3, eq. (1), (2)]. These two signals are independent from each other or uncorrelated. The Rayleigh signals, $E_{x}$ and $E_{y}$, can be complex Gaussian signals so therefore another representation of the $E$-fields is:

$$
\begin{aligned}
& E_{x}=r_{1} e^{j\left(\omega t-\phi_{1}\right)} \\
& E_{y}=r_{2} e^{j\left(\omega t-\phi_{2}\right)}
\end{aligned}
$$

By taking the square envelopes of $V_{1}$ and $V_{2}$ to be $R_{1}$ and $R_{2}$ respectively (i.e. half the square magnitude of the voltage) then:

$$
\begin{aligned}
& 2 R_{1}^{2}=a^{2} e^{2} r_{1}^{2}+b^{2} f^{2} r_{2}^{2}+2 r_{1} r_{2} a b e f \cos \left(\phi_{1}-\phi_{2}\right) \\
& 2 R_{2}^{2}=c^{2} e^{2} r_{1}^{2}+d^{2} f^{2} r_{2}^{2}+2 r_{1} r_{2} \text { cdef } \cos \left(\phi_{1}-\phi_{2}\right)
\end{aligned}
$$

which is in agreement with Vaughan and Kozono.

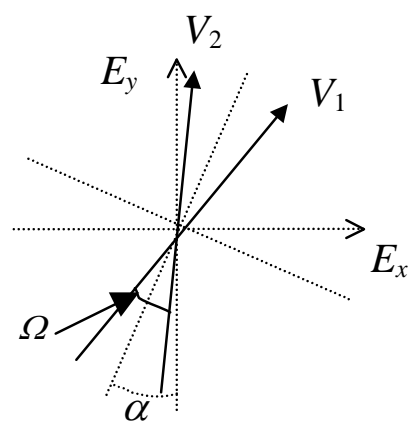

(a)
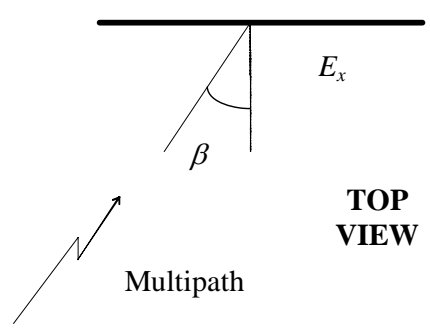

(b)

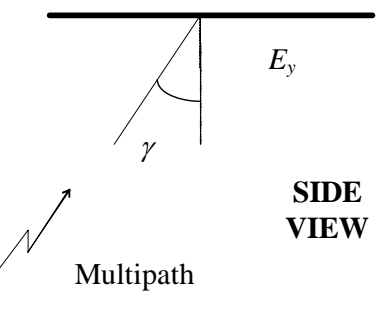

(c) Figure 1 - Diagram showing the four angles used in a
polarization diversity system

\section{A. Correlation Coefficient}

Now the E-fields are represented in complex exponential form, it is simple to derive the complex correlation coefficient, used by Adachi [10, eq. (7)]: 


$$
\rho_{12}=\frac{\overline{V_{1} V_{2}^{*}}-\overline{V_{1}} \cdot \overline{V_{2}^{*}}}{\sqrt{\overline{V_{1} V_{1}^{*} V_{2} V_{2}^{*}}}}
$$

By using equation (1), the expected voltages are:

$$
\begin{aligned}
& \overline{V_{1} V_{2}^{*}}=a c e^{2} \overline{r_{1}^{2}}+b d f^{2} \overline{r_{2}^{2}} \\
& \overline{V_{1} \cdot V_{2}^{*}}=0 \\
& \overline{V_{1} V_{1}^{*}}=a^{2} e^{2} \overline{r_{1}^{2}}+b^{2} f^{2} \overline{r_{2}^{2}} \\
& \overline{V_{2} V_{2}^{*}}=c^{2} e^{2} \overline{r_{1}^{2}}+d^{2} f^{2} \overline{r_{2}^{2}}
\end{aligned}
$$

then the resultant complex correlation coefficient at any given three dimensional angle for two given polarizations (always real in this case) is:

$$
\rho_{12}=\frac{\left(\tan \left(\alpha-\frac{\Omega}{2}\right) \tan \left(\alpha+\frac{\Omega}{2}\right) \cos ^{2} \beta+\mathrm{XPR} \cos ^{2} \gamma\right)}{\sqrt{\left(\tan ^{2}\left(\alpha-\frac{\Omega}{2}\right) \cos ^{2} \beta+\mathrm{XPR} \cos ^{2} \gamma\right)\left(\tan ^{2}\left(\alpha+\frac{\Omega}{2}\right) \cos ^{2} \beta+\mathrm{XPR} \cos ^{2} \gamma\right)}}
$$

where XPR is the time averaged cross-polar power ratio of the received field (and not of the antennas) [8] [14], defined as:

$$
\mathrm{XPR}=\frac{\overline{r_{2}^{2}}}{\overline{r_{1}^{2}}}
$$

This notation has been previously described by Vaughan and Kozono as cross-polar discrimination (XPD). It has not been used here since it is then not contrary to the definition of XPD explained by Allnutt [5], which is dependent on the axial ratio (AR). Therefore XPR has been chosen so that it is identified as a time averaged power ratio applicable to evaluating correlation and mean effective gain.

Another point to note is that the power correlation (that can be assumed equal to the envelope correlation, $\rho_{e}$, [6]) when derived using $R_{1}$ and $R_{2}$ as done by Vaughan and Kozono matches the Clarke approximation [11] below:

$$
\rho_{e}=\left|\rho_{12}\right|^{2}
$$

If equation (17) is substituted into equation (19) then it is in agreement with equations used by both Vaughan and Kozono. If $\alpha=0^{\circ}$ and $\gamma=0^{\circ}$ then the equation reduces to Kozono's. Likewise, if $\alpha$ is shifted by $45^{\circ}$ and $\Omega, \beta$ and $\gamma=0^{\circ}$, then the equation reduces to Vaughan's as would be expected.

Measurements suggest a value of $6 \mathrm{~dB}$ to be the average in an urban fading environment [4], although this value refers to the base station case. Cross-polar ratio has not yet been measured fully at the mobile so it is assumed to be $6 \mathrm{~dB}$ here. With this in mind, equations (17) and (19) can be applied to a suitable angle of arrival (AOA) model at the mobile which is presented in equation (20). The angle of arrival is defined by the probability density functions, $p(\beta)$ and $p(\gamma)$. For many years it has been difficult to characterize the AOA due to the limits of measuring antennas. Many different models have been proposed as discussed by Ertel [7]. An appropriate mathematical model to evaluate the mean effective gain (MEG) is proposed by Taga [8]. In this model the distribution is uniform in azimuth (since there are random scatterers around the mobile) and Gaussian in elevation (since the base station is above the mobile without many scatterers at different heights above the mobile). Measurements made by Taga indicate that the mean AOA for the Gaussian elevation distribution is around $20^{\circ}$ above the horizontal and also the standard deviation varies around $20^{\circ}$. Therefore these quantities will be used for analysis purposes. It must be noted though that this applies only to an outdoor Rayleigh fading environment. By applying the angle of arrival model, the correlation can be evaluated for every value of $\beta$ and $\gamma$, translated from $\theta$ and $\phi$ in [12, eq. (8)]:

$$
\begin{aligned}
& \overline{\rho_{e}}=\frac{\left|\int_{-\pi}^{0} \int_{-\frac{\pi}{2}}^{\frac{3 \pi}{2}}\left(a c e^{2}+b d f^{2} \mathrm{XPR}\right) p(\beta) p(\gamma) \cos \gamma d \beta d \gamma\right|^{2}}{\left|\int_{-\pi} \int_{-\frac{\pi}{2}}^{\frac{3 \pi}{2}}\left(a^{2} e^{2}+b^{2} f^{2} \mathrm{XPR}\right) p(\beta) p(\gamma) \cos \gamma d \beta d \gamma\right|^{2}} \times \\
& \left|\int_{-\pi}^{0} \int_{-\frac{\pi}{2}}^{\frac{3 \pi}{2}}\left(c^{2} e^{2}+d^{2} f^{2} \mathrm{XPR}\right) p(\beta) p(\gamma) \cos \gamma d \beta d \gamma\right|^{2}
\end{aligned}
$$

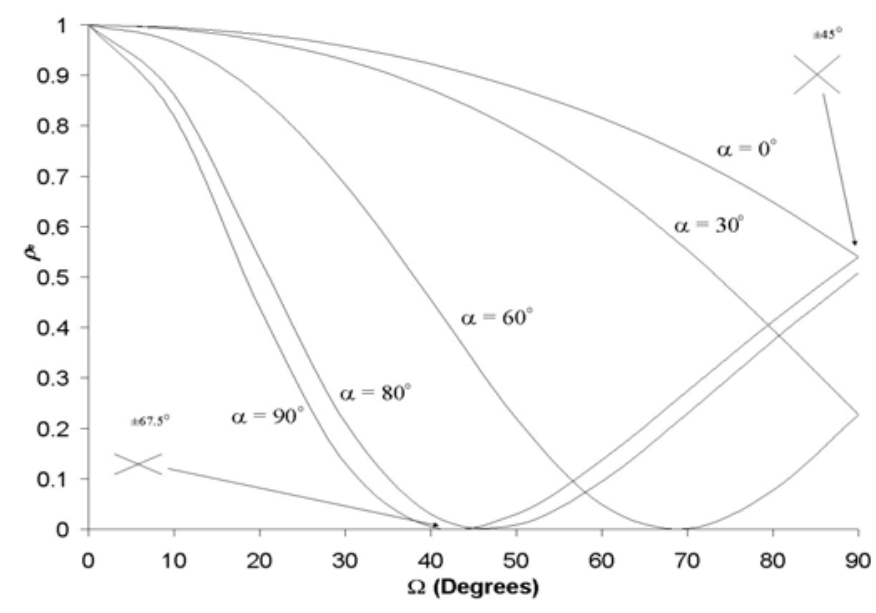

Figure 2 - Graph showing the correlation in different polarizations at the mobile with the angle of arrival applied

Equation (20) is plotted in Figure 2 where the correlation is given versus angle $\Omega$ for fixed values of $\alpha$. The graph shows that there are a number of points where there is minimum correlation; the most notable, as will be seen later, is where $\alpha$ 
is $90^{\circ}$ and $\Omega$ is around $45^{\circ}$, which means the polarizations in space have a $\pm 67.5^{\circ}$ configuration.

\section{B. Branch Power Ratio}

Maximum diversity gain does not necessarily occur at points where there is minimum correlation. The branch signal level difference, $k$, between the two antennas may conflict with this. Derivation of $k$ is as follows using equations (10) and (11) if the angle of arrival is not considered.

$$
k=\frac{\overline{R_{2}^{2}}}{\overline{R_{1}^{2}}}=\frac{\cos ^{2} \beta+\mathrm{XPR} \cos ^{2} \gamma+\cos (2 \alpha-\Omega)\left(\mathrm{XPR} \cos ^{2} \gamma-\cos ^{2} \beta\right)}{\cos ^{2} \beta+\mathrm{XPR} \cos ^{2} \gamma+\cos (2 \alpha+\Omega)\left(\mathrm{XPR} \cos ^{2} \gamma-\cos ^{2} \beta\right)}
$$

Equation (21) can be applied in the same manner as in equation (20) with correlation to determine an averaged value of $k, \bar{k}$.

\section{Polarization Diversity Gain and System Gain}

Correlation and branch power ratio can be applied to find their effects on diversity gain [6] although when diversity gain is considered it has to be carefully defined at the mobile as it may not necessarily always reflect the performance of the selection diversity receiver, as will be seen. At the base station, the diversity gain is a comparison of the mean signal to noise ratio of two systems at a given reliability level; 99.9\% is used in this instance. Traditionally, in the case of a base station, the single branch reference has been the vertical polarization since that is what would otherwise be present in a non-diversity system. Therefore we need to apply the loss factor, $L$, in a similar way to Kozono [3, eq. (15)], which in this instance is used for a selection diversity combiner rather than a maximum ratio combiner:

$$
L=\max \left[\frac{\overline{R_{1}^{2}}}{\overline{R_{o}^{2}}}, \frac{\overline{R_{2}^{2}}}{\overline{R_{o}^{2}}}\right]
$$

where:

$$
\frac{\overline{R_{1,2}^{2}}}{\overline{R_{0}^{2}}}=\frac{\sin ^{2}\left(\alpha \pm \frac{\Omega}{2}\right) \cos ^{2} \beta+\mathrm{XPR} \cos ^{2}\left(\alpha \pm \frac{\Omega}{2}\right) \cos ^{2} \gamma}{\sin ^{2} \alpha_{0} \cos ^{2} \beta+\mathrm{XPR} \cos ^{2} \alpha_{0} \cos ^{2} \gamma}
$$

where $\overline{R_{0}^{2}}$ defines the reference polarization at a given angle, $\alpha_{0}$. At the base station, this would be normally the vertical, when $\alpha_{0}$ is zero but at the mobile terminal, however, the vertical is not necessarily a good reference as far as talk position or other positions are concerned. The reference used is arbitrary, although it must be clearly defined and kept consistent when comparing different systems. In this paper $60^{\circ}$ has been chosen (i.e. $30^{\circ}$ above the horizontal).

Like correlation and branch power ratio, $k$, equations (22) and (23) can be applied to determine $\bar{L}$ averaged over the angle of arrival distribution to determine the system gain which is related to diversity gain by the following equation:

$$
\text { System Gain }=\text { Diversity Gain } \times \bar{L}
$$

Careful note needs to be made here regarding the distinction between system gain and diversity gain at the mobile because if a gain is seen, it is not necessarily diversity gain. This is best illustrated in Figure 3 where system gain is plotted in the same way as correlation in Figure 2. It is important to note that there are values of gain or loss even when $\Omega$ is zero and correlation, $\rho_{12}$, is unity in this instance. This is due to $L$ not being unity where diversity gain is $0 \mathrm{~dB}$ (because there is unity correlation) and system gain is not. Therefore, it is wrong to say here there is diversity gain, when the diversity combiner is making no difference, it is inactive. System gain is therefore a more appropriate term to account for this.

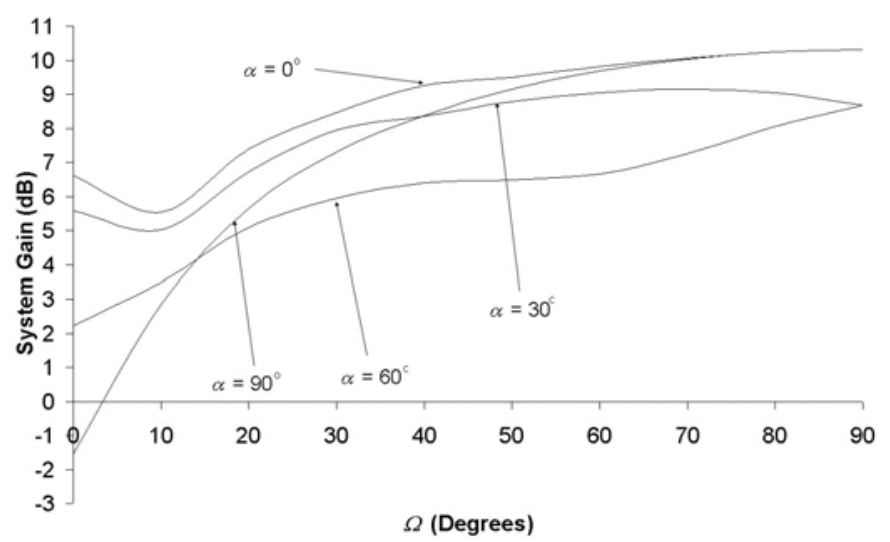

\section{Figure 3 - Graph showing the system gain in different polarizations at the mobile with the angle of arrival applied}

It is noted from Figure 3 that the best overall system gain occurs at the $\pm 45^{\circ}$ (or $\alpha=45^{\circ}, \Omega=90^{\circ}$ ) configuration as is typical for polarization diversity. In Figure 2, however, this is not seen to be the case where other configurations like $\pm 67.5^{\circ}$ give an improved de-correlation, where there is optimum diversity gain but not necessarily system gain. It is therefore important to note the correlation and branch power ratio as well as the system gain output in a fading environment are analyzed also to ensure that the selection combiner is providing optimum performance.

\section{Discussion of effects of cross polar ratio and angle of arrival}

For polarization diversity, as much cross polarization as possible (i.e. XPR is as low as possible) is always desirable so a reduction below the assumed $6 \mathrm{~dB}$ will increase the diversity potential and also move the optimum positioning of the two polarizations. In suburban and rural environments, XPR is likely to increase and so there is a maximum XPR to achieve polarization diversity. For XPR above $15 \mathrm{~dB}$ there is little scope for polarization diversity in order to achieve suitable decorrelation, $\rho_{12}$, a reasonable branch power ratio, $k$, and signal to noise ratio loss, $L$. It is therefore necessary that a suitable level of scattering is available to gain sufficient depolarization from a single polarization source. 


\section{E. Effect of angle of arrival}

Considering measurements undertaken by Taga [8], the main variation when using the Gaussian model for the elevation angle of arrival is that the standard deviation rather than the mean is changing to any significant degree. When analyzing the correlation and overall system gain, analysis does not show any significant change so the results that are seen in Figure 2 and Figure 3 do not change to any significant degree. Using the model proposed in this paper therefore, the effects of changing angle of arrival in normal cases are insignificant.

\section{TheOretical Model For MEAsuring POLARIZATION AT THE MOBILE}

This section pays attention to the affects of a measuring antenna if the polarization characteristics of a fading environment were ever to be measured. Note here this is only considering measurement of the fading environment in isolation, not the polarization diversity antennas themselves, which will be considered in the next section. When measuring the polarization characteristics, it is only possible to do so with a measurement antenna that has purely vertical polarization and one that has purely horizontal polarization. Crossed dipoles would not be suitable in this scenario since the horizontal dipole will still have some $E_{\theta}$ polarization as well as $E_{\phi}$ polarization so it would not be $100 \%$ horizontally polarized in this sense. A vertical dipole and a horizontal loop antenna would be suitable since the loop has only $E_{\phi}$ polarization [14] so it can be considered a completely horizontally polarized "dipole”. Using such antennas, there are three possible effects from the measurement antenna properties that could impact on measurements as follows:

1. The complex isolation between the measurement antennas.

2. Measurement antenna impedance differences and mismatches.

3. Polarization impurities in each measurement antenna branch.

The effects of isolation and measurement antenna impedance differences can be considered by adding a transfer matrix, $\mathbf{S}_{T}$, to equation (1) as follows:

$$
\mathbf{V}_{L}=\left(\begin{array}{c}
V_{1} \\
V_{2}
\end{array}\right)=\mathbf{S}_{T}\left(\begin{array}{cc}
a e & b f \\
c e & d f
\end{array}\right)\left(\begin{array}{c}
E_{x} \\
E_{y}
\end{array}\right)
$$

where $\mathbf{S}_{T}$, is derived from Vaughan and Bach Andersen [13] applied to a two-port impedance network for measurement antenna impedance, $\mathbf{Z}_{A}$ and load impedance $\mathbf{Z}_{L}$ (with impedance $Z_{0}$ at each terminal):

$$
\mathbf{S}_{T}=\mathbf{Z}_{A}\left(\mathbf{Z}_{A}+\mathbf{Z}_{L}\right)^{-1}
$$

Using two-port $\mathbf{S}$-parameter conversions [15] the transfer matrix is best expressed in terms of S-parameters (assuming a reciprocal network if $s_{12}=s_{21}$ ):

$$
\mathbf{S}_{T}=\left(\begin{array}{cc}
\frac{s_{21}\left(z_{22}+Z_{0}\right)}{2 z_{21}} & -\frac{s_{12}}{2} \\
-\frac{s_{21}}{2} & \frac{s_{12}\left(z_{11}+Z_{0}\right)}{2 z_{12}}
\end{array}\right)=\left(\begin{array}{cc}
s_{f 1} & s_{r} \\
s_{r} & s_{f 2}
\end{array}\right)
$$

It is useful to note that $\mathbf{S}_{T}$ is determined by isolation, $\mathbf{s}_{r}{ }^{-1}$, and load transfer at each port, $s_{f 1}$ and $s_{f 2}$. If the measurement antennas are identical then $s_{f 1}=s_{f 2}=s_{f}$. Further to this, if the ports are matched then $s_{f}=0.5$. After applying $\mathbf{S}_{T}$, the complex correlation at each angle as in equation (17) becomes:

$$
\rho_{12}=\frac{\left.\left[s_{f 1} a+s_{r} c\right]\right] s_{f 2}^{*} c+s_{r}^{*} a e^{2}}{\sqrt{\left(\mid s_{f 1} b+s_{r} d\right]\left[s_{f 2}^{*} d+s_{r}^{*} b\right] f^{2} \mathrm{XPR}}}
$$

Equation (28) indicates that there could be an imaginary component present in the measured correlation with low isolation, $s_{r}^{-1}$ (i.e. $s_{12}$ is close to unity) or impedance mismatch. If there is high isolation (i.e. low $s_{12}$ ) it reduces to equation (17). The branch difference, $k$, and overall signal loss, $L$, will be affected by $\mathbf{S}_{T}$ so they change as follows:

$$
\begin{aligned}
& k=\frac{\left|s_{f 2} c+s_{r} a\right|^{2} e^{2}+\left|s_{f 2} d+s_{r} b\right|^{2} f^{2} \mathrm{XPR}}{\left|s_{f 1} a+s_{r} c\right|^{2} e^{2}+\left|s_{f 1} b+s_{r} d\right|^{2} f^{2} \mathrm{XPR}} \\
& L=\frac{\max \left(\left|s_{f 1} a+s_{r} c\right|^{2} e^{2}+\left|s_{f 1} b+s_{r} d\right|^{2} f^{2} \mathrm{XPR},\right.}{\left.\left|s_{f 2} c+s_{r} a\right|^{2} e^{2}+\left|s_{f 2} d+s_{r} b\right|^{2} f^{2} \mathrm{XPR}\right)} \\
& \overline{R_{0}^{2}}
\end{aligned}
$$

Equations (29) and (30) reduce to (21) and (23) with high isolation, $s_{r}{ }^{-1}$, and with impedance matching. Another factor is that the measurement antennas may have the same polarization impurity, $\mathbf{M}$, defined as follows:

$$
\mathbf{M}=\left(\begin{array}{cc}
1 & V_{X P} / V_{C P} \\
V_{X P} / V_{C P} & 1
\end{array}\right)=\left(\begin{array}{cc}
1 & m \\
m & 1
\end{array}\right)
$$

where $V_{C P}$ and $V_{X P}$ are the co and cross-polar complex voltages of the measurement antennas respectively. Therefore $m$ is defined as a complex voltage ratio. When $\mathbf{M}$ is multiplied by $\mathbf{S}_{T}$ equation (30) can be further modified to include polarization impurities: 


$$
\rho_{12}=\frac{+\left[s_{f 1}(b+a m)+s_{r}(d+c m)\right]\left[s_{f 2}^{*}\left(d+c m^{*}\right)+s_{r}^{*}\left(b+a m^{*}\right)\right] f^{2} X P R}{\sqrt{\left(\left|s_{f 1}(a+b m)+s_{r}(c+d m)\right|^{2} e^{2}+\left|s_{f 1}(b+a m)+s_{r}(d+c m)\right|^{2} f^{2} X P R\right)}}
$$

It must be further noted that the two measurement antennas may have different polarization impurities $\left(m_{1}\right.$ and $\left.m_{2}\right)$, and that they may vary between angles, which causes equation (32) to be even more complex and have further effects on measurement. For low values of complex isolation $\left(s_{r}{ }^{-1}<\right.$ $20 \mathrm{~dB}$ ), the complex correlation can result in having an imaginary part along with reduction in diversity gain. Also shown by the simulations is that high polarization impurity above $-20 \mathrm{~dB}$ will degrade diversity gain by more than $1 \mathrm{~dB}$, which is in agreement with Collins [9]. Further reduction comes with two measurement antennas with a high difference in gain ( $>3 \mathrm{~dB}$ ). This is mainly due to the branch difference, $k$, becoming too high.

It should be noted for the above cases, that it is only possible to measure the two polarizations with realizable measurement antennas using the vertical/horizontal (i.e. orthogonal) configuration so $a=1, b=0, c=-1$ and $d=0$. It is unfortunately not possible to implement antennas to measure the non orthogonal polarizations considered in this model. This therefore also means that polarization diversity antennas that take full advantage of the polarization diversity present in the fading environment cannot be deployed. Therefore, by noting this point, a new model is described in the next section which will show the distinctive difference between polarization diversity of the fading environment and polarization diversity that is inherent within an angular diversity system.

\section{Antenna Polarization Diversity at the MOBILE}

Up to this section, only polarization diversity of the fading environment between two polarizations in space at the mobile has been considered. This final section will show how there is a distinction between the antenna polarization diversity and the polarization diversity of the fading environment, both of which have different applications. The method proposed in this section shows how two antennas that have angular diversity may also have differing polarizations so that there is a contribution of polarization diversity inherent within the angular diversity system. For mobile antennas that do not have a directional antenna and a narrow angle of arrival, as at the base station, this is the only realistic way to evaluate polarization diversity where the $A_{\theta}$ and $A_{\phi}$ polarizations of the antenna have to be considered at each angle as illustrated in Figure 4 and then they can be compared to the total polarization also shown. To correlate the polarizations of two diversity antennas it is possible to apply equation (33) from [16 eq. (28)] to evaluate the correlation between two different antenna polarizations. This equation is able to show that a pure polarization diversity antenna such as a horizontal loop and vertical dipole antenna will have zero angular correlation with zero polarization correlation because angular diversity is a comparison of angular patterns in the same polarization [16 eq. (23)]. To show what polarization contribution there is in two examples of polarization diversity antennas there are two scenarios in Figure 5 where the angular spacing is varied between the two dipoles but with different orientations. Firstly, Figure 6 shows that when angle $\varphi$ is opened out to $90^{\circ}$, there is not zero polarization correlation in the antennas because the horizontal dipole has some $A_{\theta}$ components as well as $A_{\phi}$ components so therefore there is not total polarization de-correlation unlike the fading environment and the angular de-correlation shown in Figure 6 also. The same analysis is shown for Figure 5 (b) in Figure 7 also with a different orientation for angle $\varepsilon$, where there is little polarization decorrelation within the antennas. It is therefore clear that changing the polarizations between two dipoles is creating an angular diversity system with some inherent polarization diversity also and that the polarization diversity of two polarizations in space is a completely separate matter not related to that of the antennas.

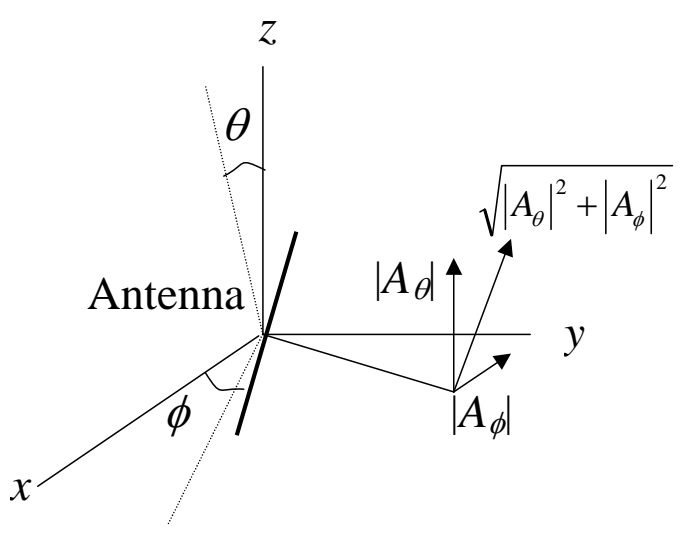

\section{Figure 4 Diagram showing the $A_{\theta}$ and $A_{\phi}$ polarizations and total polarization of an antenna at a given angle}

To evaluate the total diversity gain and system gain of the two polarization diversity antennas at different polarizations, it is necessary to evaluate their mean effective gain [8] from which branch power ratio, $k$, and signal gain/loss $L$ could be derived by taking appropriate ratios of the evaluated mean effective gains. Only analysis of the correlation, however, has been presented here because it shows what contributions of polarization diversity there are in two mobile terminal antennas if there is any system gain. A further factor not considered in this scenario is the mutual coupling effects of having the two dipoles in the presence of each other, where their field patterns will be distorted. This has not been considered as it is not so practical to have two co-located dipoles in the presence of each other and measure or simulate their field patterns. Where any two simulated or measured diversity antennas are evaluated in this way, the mutual coupling which will contribute to the angular and polarization 
diversity will be inherent in the evaluation. It is also possible that the presence of the user or other nearby objects can be included if necessary.

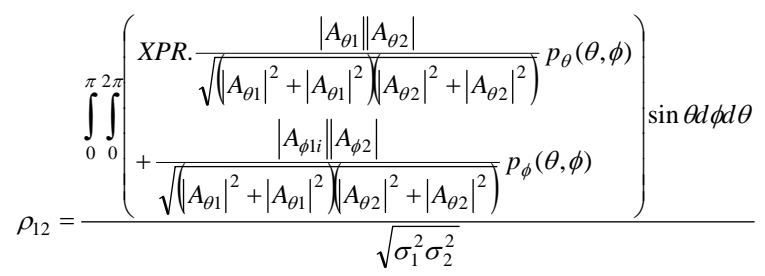

$$
\sigma_{n}^{2}=\int_{0}^{\pi} \int_{0}^{2 \pi}\left(X P R \cdot \frac{\left|A_{\theta n}\right|^{2}}{\left|A_{\theta n}\right|^{2}+\left|A_{\theta n}\right|^{2}} p_{\theta}(\theta, \phi)+\frac{\left|A_{\phi n}\right|^{2}}{\left|A_{\theta n}\right|^{2}+\left|A_{\theta n}\right|^{2}} p_{\phi}(\theta, \phi)\right) \sin \theta d \phi d \theta
$$

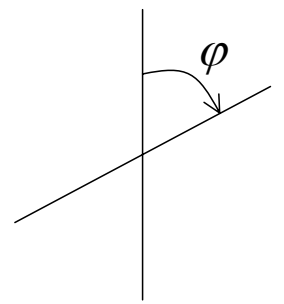

(a)

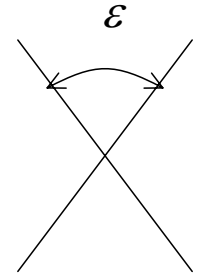

(b)
Figure 5 - Comparison of two antenna polarizations to be applied to polarization diversity

\section{CONCLUSION}

A new theoretical model for polarization diversity at the mobile terminal has been discussed where the differences between polarization diversity of a fading environment and the polarization diversity inherent within an angular antenna diversity system are distinguished. Results show that for two polarizations in space (not that of the antennas) the best output with maximum system gain is at the $\pm 45^{\circ}$ configuration although there is minimum correlation and equal branch power at $\pm 67.5^{\circ}$, where the selection diversity combiner will perform best and give optimum diversity gain. This is important to note so it is understood how well the diversity system is performing internally. Attention is also given to characterizing the polarizations at the mobile using a loop and dipole antenna and thus showing how a new modeling approach is necessary at the mobile.

Taking two dipoles as an example, the second model approach has shown how much polarization diversity contribution there is when evaluating the angular diversity of two ideal dipole antennas as an example. The difference between this and the polarization diversity of a fading environment between two polarizations in space is explained. Results show that dipoles and other linear antennas can have some, although not always a large degree of polarization diversity if they do have different polarization orientations.

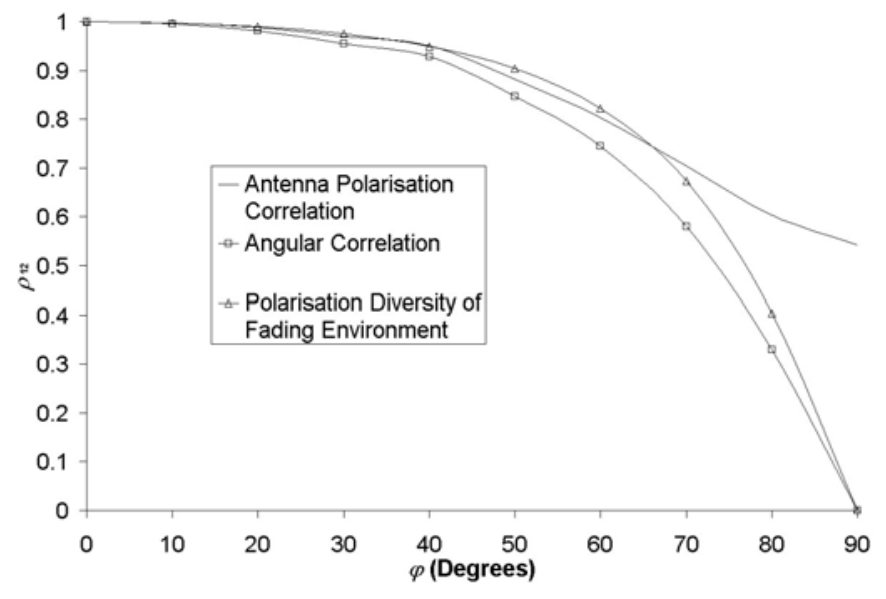

Figure 6 - Comparison of antenna polarization correlation, angular correlation and fading environment polarization correlation for the scenario in Figure 5 (a)

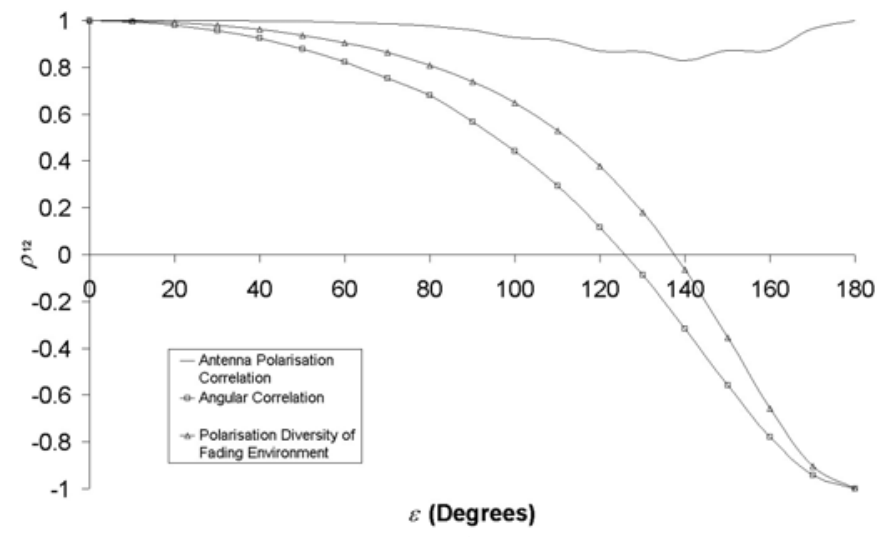

Figure 7 - Comparison of antenna polarization correlation, angular correlation and fading environment polarization correlation for the scenario in Figure 5 (b)

\section{ACKNOWLEDGMENT}

Many thanks goes to the support of Nokia Mobile Phones who were sponsors of this work.

\section{REFERENCES}

[1] C. Beckman, U. Wahlberg, “Antenna Systems for Polarization Diversity”, Microwave Journal, May 1997, pp330-334.

[2] R.G. Vaughan "Polarization Diversity for Mobile Communications", IEEE Trans Vehicular Technology, vol. VT-39, no. 3, pp177-186, August 1990.

[3] S. Kozono, T.Tsuruara, M Sakamoto, "Base Station Polarization Diversity for Mobile Radio", IEEE Trans Vehicular Technology, vol. VT-33, no. 4, pp301-306, November 1984.

[4] W. C. Y. Lee, Y. S. Yeh, "Polarization Diversity System for Mobile Radio”, IEEE Trans Communications, vol. COM-20, no. 5, pp912-923, October 1972.

[5] J. E. Allnutt, Satellite to Ground Radiowave Propagation, 1989, IEE Peter Pergrinus Ltd, pp11-13

[6] M. Schwartz, W. Bennett, S. Stein, Communication Systems and Techniques, 1966, McGraw Hill Inc, pp416-489.

[7] R. B. Ertel, K. W. Sowerby, T. S . Rappaport, "Overview of Spatial Channel Models for Antenna Array Communication Systems”, IEEE Personal Communications, pp10-22, Feburary 1998. 
[8] T. Taga, "Analysis of Mean Effective Gain of Mobile Antennas in Land Mobile Radio Environments”, IEEE Trans Vehicular Technology, vol VT-39, no 2, pp117-131, May 1990.

[9] B. S. Collins, "The Effect of Imperfect Antenna Cross-Polar Performance on the Diversity Gain of a Polarization-Diversity System", Microwave Journal, pp43-49, April 2000.

[10] F. Adachi, M.T. Feeney, A.G. Williamson, J.D. Parsons, "Crosscorrelation between the envelopes of $900 \mathrm{MHz}$ signals received at a mobile radio base station site”, IEE Proc Radar and Signal Processing, vol. 133, no. 6, Part F, Oct 1986, pp506-512.

[11] R. H. Clarke, “A Statistical Theory of Mobile Radio Reception”, Bell System Technical Journal, 1968, no. 47, pp957-1000.

[12] G. F. Pedersen, J. Bach Andersen, "Handset Antennas for Mobile Communications: Integration, Diversity and Performance”, Review of Radio Science, 1996, pp119-139.

[13] R. G. Vaughan, J. Bach Andersen, "Antenna Diversity in Mobile Communications”, IEEE Trans Vehicular Technology, vol. 36, no. 4, November 1987, pp149-172.

[14] D. C. Cox, R. R. Murray, H. W. Arnold, A. W. Norris, M. F. Wazowicz, "Cross Polarization and Coupling Measurements for $800 \mathrm{MHz}$ Radio Transmission in and around Houses and Large Buildings", IEEE Transactions on Antennas and Propagation, vol. 34 no. 1, January 1986, pp83-87.

[15] G. G. Johnstone, J. H. B. Deane, "Relations between two-port parameters", International Journal of Electronics, vol. 71, no. 1, December 1990, pp107-116.

[16] T. W. C. Brown., S. R. Saunders, B. G. Evans, "Analysis of Mobile Terminal Diversity Antennas”, IEE Proceedings, Microwaves, Antennas and Propagation, vol. 152, February 2005, pp1-6.

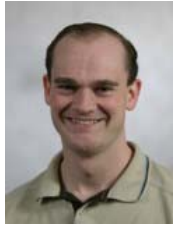

Tim W. C. Brown (S'00-M'02) received his BEng degree in Electrical and Electronic Engineering in 1999 and subsequently gained his $P h D$ in 2002 both at the Centre for Communication Systems Research (CCSR) University of Surrey, UK. As well as working at Surrey Tim has been working for over 2 years within the Antennas, Propagation and Networking Group at Aalborg University, Denmark as an Assistant Research Professor up to the end of 2006 and recently taken up the position as a lecturer back at CCSR with specialization in physical layer channel characterization and antennas for mobile terminals. Tim's research interests specialize in multi-element antenna communications with particular interest in diversity antennas, intelligent adaptive antenna concepts and multiple input multiple output (MIMO) systems.

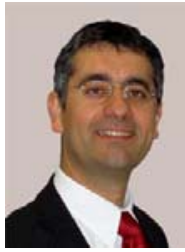

Simon R. Saunders (S'89-M'91) has specialised in wireless communications throughout his career, with particular interests in radio wave propagation prediction in urban and indoor environments, smart antennas, and mobile systems engineering. He has pursued these interests in both industry and academia. Simon is Chief Technology Officer for the Red-M Group, developing and delivering services and products via a best-practice approach which he originated, known as "Total Airspace Management". He is also a Visiting Professor at the University of Surrey. Simon has acted as a consultant to a wide range of companies, including BAA, BBC, Mitsubishi, British Land, O2, Ofcom, BT, ntl and many others.

Prof. Saunders has invented more than fifteen patented technologies and has written more than 130 publications in learned journals and international conferences. He has also written a successful book, "Antennas and Propagation for Wireless Communication Systems", which will appear in a second edition shortly.

He obtained BSc (Hons) and PhD degrees from Brunel University in 1988 and 1991 respectively, receiving four separate awards for academic achievement in that time. He worked on microwave oscillator structures as a Research Fellow for Brunel in 1991. In 1993-4, Prof. Saunders proposed a novel parallel computing approach to the solution of electromagnetic problems and acted as consultant to the resulting project, which led to the creation of an electromagnetic simulation package, used as a CAD and teaching tool.
For several years he worked in an advanced development role for Philips Telecom, including the standardisation of the TETRA air interface for digital Private Mobile Radio. He led a team designing technology for a novel twoway paging system. Simon also spent one year working with the Ascom in Switzerland, defining product technologies and system design solutions for the first generation of TETRA handsets and base stations.

Prof. Saunders worked for Motorola in their GSM Research Group in 1995, designing air interface and system planning enhancements leading to increased capacity second generation GSM systems. This included several patented advances in antenna, modem and cellular architectures.

Simon lectured on mobile systems and led a research team within the Centre for Communication Systems Research at The University of Surrey for seven years. He proposed and delivered many research programmes for industry and government research councils and acted as Director of Continuing Education.

As Chief Technology Officer and then Chief Executive Officer of Cellular Design Services Ltd., Simon developed a suite of software tools and services for auditing and planning a wide variety of radio systems and won a major contract to act as the Radio Strategy Partner to BAA, the UK's leading airports authority. He led the company into a merger with Red-M Communications and raised development funds to enable the company's growth and diversification.

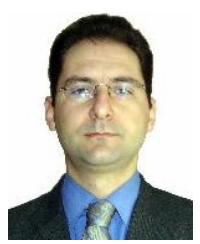

Stavros Stavrou was born in Nicosia, Cyprus, on March 10, 1972. He received a diploma in Electrical engineering from Higher Technical Institute in Cyprus in 1992. In 1996 he was awarded the BEng degree in Communications and Computer engineering from the University of Essex, UK and in 2001 the $P h D$ degree in Electronic and Electrical Engineering from the University of Surrey, UK. His PhD work concentrated on radiowave propagation and RF design for mobile communications. From 1997-2000 he was a research fellow with the Centre for Communication Systems Research (CCSR), University of Surrey, UK. During this period he worked on several national and international research projects. In 2000 he was appointed as a lecturer, at CCSR, at the University of Surrey. His main research interests include radiowave propagation, MIMO, UWB systems and radio frequency and antenna design. Dr Stavrou has authored and co authored a number of papers in the above areas.

Mauro Fiacco graduated from University of Westminster, London, in 1994 in

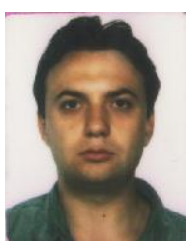
Electronic Engineering. He subsequently worked at Motorola Ltd, developing Layer 1 and Layer 2 software for GSM base stations. He has completed a Ph.D. at at the University of Surrey in 2001; where his research interests included radio propagation in indoor environment and radio architecture for future generation communication systems. Currently, he is research engineer for ip.access Ltd (UK), his research interests include network time protocols and still carry out work on propagation studies for second and third generation telecommunication systems. 